\title{
Circumferential resonance modes of solid elastic cylinders excited by obliquely incident acoustic waves
}

\author{
Ying Fan \\ Department of Mechanical and Industrial Engineering, University of Toronto, 5 King's College Road, \\ Toronto, Ontario M5S 3G8, Canada \\ Farhang Honarvar \\ Department of Mechanical Engineering, K. N. Toosi University of Technology, P.O. Box 16765-3381, \\ Tehran, Iran \\ Anthony N. Sinclair ${ }^{a)}$ \\ Department of Mechanical and Industrial Engineering, University of Toronto, 5 King's College Road, \\ Toronto, Ontario M5S 3G8, Canada \\ Mohammad-Reza Jafari \\ Department of Mechanical Engineering, K. N. Toosi University of Technology, P.O. Box 16765-3381, \\ Tehran, Iran
}

(Received 21 March 2002; revised 5 October 2002; accepted 7 October 2002)

\begin{abstract}
When an immersed solid elastic cylinder is insonified by an obliquely incident plane acoustic wave, some of the resonance modes of the cylinder are excited. These modes are directly related to the incidence angle of the insonifying wave. In this paper, the circumferential resonance modes of such immersed elastic cylinders are studied over a large range of incidence angles and frequencies and physical explanations are presented for singular features of the frequency-incidence angle plots. These features include the pairing of one axially guided mode with each transverse whispering gallery mode, the appearance of an anomalous pseudo-Rayleigh in the cylinder at incidence angles greater than the Rayleigh angle, and distortional effects of the longitudinal whispering gallery modes on the entire resonance spectrum of the cylinder. The physical explanations are derived from Resonance Scattering Theory (RST), which is employed to determine the interior displacement field of the cylinder and its dependence on insonification angle. (C) 2003 Acoustical Society of America.
\end{abstract} [DOI: 10.1121/1.1525289]

PACS numbers: 43.20.Fn, 43.40.Ks [ANN]

\section{INTRODUCTION}

An analysis of the ultrasonic scattering characteristics of a cylinder may be used to determine its geometric and elastic properties, or the boundary conditions between the cylinder and the surrounding medium. ${ }^{1,2}$ The scattered pressure field from a submerged cylinder contains valuable information about the resonance modes of the cylinder. The resonance modes of the cylinder serve as its signatures, therefore, it is necessary to have a thorough understanding of the characteristics of these resonance modes in order to study the properties of the cylinder. In this paper, the dependence of the scattering spectrum and resonance modes of an immersed solid elastic cylinder insonified by a plane acoustic wave is explored as a function of the incidence angle of the insonifying wave. Physical explanations for the dependence of the resonance frequencies on the angle of incidence are given. The dependence of the resonance frequencies on the angle of incidence has never been studied to this extent before.

Earliest studies of wave scattering from cylinders, conducted by Faran, dealt with normally incident compressional waves on a submerged elastic, isotropic, and homogeneous

\footnotetext{
a) Author to whom correspondence should be addressed. Electronic mail: sinclair@mie.utoronto.ca
}

rod. ${ }^{3}$ A normal mode expansion technique was used; this technique has now become relatively straightforward with the advent of high-powered desktop computers and mathematical libraries such as $\mathrm{IMSL}^{4}$ and MATLAB. ${ }^{5}$ The scattering spectrum consists of a number of sharp resonances, superimposed on a relatively flat background. A physical explanation for this general form of response was provided by Resonance Scattering Theory (RST), according to which each resonance can be linked to the constructive interference of a single surface wave making multiple encirclements of the cylinder. ${ }^{6-8}$

Several researchers have explored extensions of RST to more general cases than those studied by Faran. These include investigations on the effects of obliquely incident plane waves, ${ }^{9-11}$ absorptive materials, ${ }^{12,13}$ multi-layered cylinders, ${ }^{2,13}$ encasement in a solid matrix, ${ }^{14,15}$ and material anisotropy. ${ }^{16,17}$ In each case, the method of solution follows the same format: the wave equation is solved in cylindrical coordinates in each medium, yielding a normal mode expansion with several unknown coefficients. Boundary conditions expressed in terms of stresses and displacements at each cylindrical interface are then used to solve for the coefficients. Extensive bibliographies and reviews of these topics can be found in the works by Uberall, ${ }^{8}$ Gaunaurd, ${ }^{18}$ and Addison and Sinclair. ${ }^{14}$ 


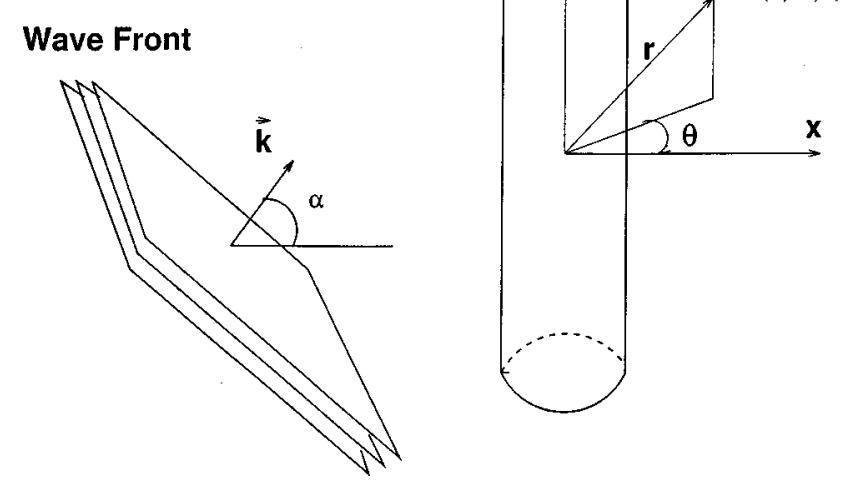

FIG. 1. Geometry used for formulating the problem.

The case of obliquely incident waves holds particular interest for a certain class of nondestructive evaluation (NDE) problems. Fibers used in reinforced composite materials typically possess transverse isotropy. Ultrasonic characterization of the axial properties of the fiber and fiber/matrix interface requires that the incident wave impinge on the cylinder at an oblique angle of $\alpha \neq 0$; see Fig. 1. Similar reasoning applies to the characterization of transversely isotropic rods such as those used in the telecommunications industry; ${ }^{19}$ the anisotropic grain structure and stiffness properties of these rods are closely linked to their low-noise signal transmission capabilities. ${ }^{20}$

To date, a number of RST studies have been conducted that focus on the issue of plane waves incident at oblique angles. White's formulation for wave scattering from an embedded rod provided solutions for nonzero values of $\alpha$, however, the lack of computing power available at that time restricted his numerical solutions to cases with $\alpha=0 .{ }^{21}$ Many other researchers including Flax et al., ${ }^{9}$ Veksler, ${ }^{11} \mathrm{Nagl}$ et al. ${ }^{22}$ and Maze et al. ${ }^{23}$ pursued this topic and demonstrated that beside the two classes of surface waves observed at normal incidence, i.e., Rayleigh and whispering gallery waves, an entirely new set of surface waves, termed axially guided waves, are introduced when the incident wave vector has a nonzero component along the axis of the cylinder. These waves travel along helical paths with the helix angle dependent on the phase velocity of the wave, and generate a new set of resonant modes. Such modes are significant even in experimental studies designed to feature normally incident waves because a wave with a finite wave front does have components with nonzero projection along the axis of the cylinder. This causes guided modes to exist in almost all practical applications. Conoir et al. ${ }^{24}$ demonstrated that the resonance frequencies of these vibration modes generally shift to the right as the incidence angle $\alpha$ is increased. Lecroq et al. studied the scattering of waves from air-filled finite cylindrical shells. ${ }^{25}$ They plotted the resonance curves for a steel cylindrical shell for different resonance modes.

More recent works by Fan et al. ${ }^{15}$ and Honarvar and Sinclair ${ }^{16}$ deal with transversely isotropic rods and relatively small incident angles. Experimental verification of numerical results with $\alpha>10^{\circ}$ are difficult, as such experiments require plane waves with a very broad wavefront. However, if one is to assess accurately the extent of transverse isotropy in rods designed to have enhanced axial properties, then such studies with appreciable $\alpha$ values are essential. To learn more about the nature of surface waves and resonance modes at higher angles of incidence, one has to first consider the case of isotropic cylinders. The focus of the current work is to investigate the resonance modes of an isotropic immersed elastic cylinder over a large range of incident angles, including values beyond critical angles.

\section{THEORY}

In this section, the mathematical model for the scattering of a plane acoustic wave from an immersed elastic cylinder is briefly reviewed. Details of this mathematical model were already published by the authors. ${ }^{16}$ Although the model can be used for both isotropic and transversely isotropic cylinders, only isotropic materials are considered here.

Figure 1 shows an infinite monochromatic plane acoustic wave of frequency $\omega / 2 \pi$ incident at an angle $\alpha$ on a submerged cylinder of infinite length and radius $a$.

A cylindrical coordinate system $(r, \theta, z)$ is chosen with the $z$-direction coincident with the axis of the cylinder. The pressure $p_{i}$ of the incident plane compressional wave can be written as

$$
p_{i}=\sum_{n=0}^{\infty} \varepsilon_{n} i^{n} J_{n}\left(k_{\perp} r\right) \cos (n \theta),
$$

where $\varepsilon_{n}$ is the Neumann factor $\left(\varepsilon_{0}=1\right.$ and $\varepsilon_{n}=2$ for $n$ $\geqslant 1)$ and $J_{n}$ are the Bessel functions of the first kind of order $n, k_{\perp}=k \cos \alpha, k=\omega / c$ and $c$ is the compressional wave velocity in the surrounding fluid medium. The time dependence is suppressed.

The scattered pressure field is given by

$$
p_{s}=\sum_{n=0}^{\infty} \epsilon_{n} i^{n} A_{n} H_{n}^{(1)}\left(k_{\perp} r\right) \cos (n \theta),
$$

where $H_{n}^{(1)}$ are the Hankel functions of the first kind of order $n$ and $A_{n}$ are the unknown scattering coefficients.

The displacement vector inside the cylinder is written in terms of three scalar potential functions $\phi, \chi$, and $\psi$,

$$
\mathbf{u}=\nabla \phi+\nabla \times\left(\chi \hat{e}_{z}\right)+a \nabla \times \nabla \times\left(\psi \hat{e}_{z}\right) .
$$

In order to satisfy the equations of motion, these potential functions must be of the form,

$$
\begin{aligned}
& \phi=\sum_{n=0}^{\infty} B_{n} J_{n}\left(k_{L} r\right) \cos n \theta, \\
& \psi=\sum_{n=0}^{\infty} C_{n} J_{n}\left(k_{T} r\right) \cos n \theta, \\
& \chi=\sum_{n=0}^{\infty} D_{n} J_{n}\left(k_{T} r\right) \sin n \theta,
\end{aligned}
$$

where $B_{n}, C_{n}$, and $D_{n}$ are unknown coefficients and 


$$
k_{L}=\left(\frac{\omega^{2}}{c_{L}^{2}}-k_{z}^{2}\right)^{1 / 2} ; \quad k_{T}=\left(\frac{\omega^{2}}{c_{T}^{2}}-k_{z}^{2}\right)^{1 / 2} .
$$

$c_{L}$ and $c_{T}$ are, respectively, the bulk compressional and bulk shear wave velocities of the material and $k_{z}=k \sin \alpha$. The four unknown coefficients $A_{n}, B_{n}, C_{n}$ and $D_{n}$ can be determined by applying the four boundary conditions at the water/ cylinder interface, i.e., continuity of normal displacement and normal stress as well as nullity of the shear stresses. This will result in the following system of algebraic equations:

$$
\left(\begin{array}{cccc}
a_{11} & a_{12} & a_{13} & a_{14} \\
a_{21} & a_{22} & a_{23} & a_{24} \\
0 & a_{32} & a_{33} & a_{34} \\
0 & a_{42} & a_{43} & a_{44}
\end{array}\right)\left(\begin{array}{c}
A_{n} \\
B_{n} \\
C_{n} \\
D_{n}
\end{array}\right)=\left(\begin{array}{c}
b_{1} \\
b_{2} \\
0 \\
0
\end{array}\right) .
$$

Expressions for elements $a_{i j}$ and $b_{i}$ can be found in Ref. 16 . Equation (8) can be solved for any of the unknown coefficients using Cramer's rule. By solving Eq. (8) for $A_{n}$, the scattered pressure field can be completely determined.

Resonance peaks occur at frequencies where the matrix of Eq. (8) becomes singular, i.e., its determinant becomes equal to zero. The roots of this determinant are the circumferential resonance frequencies of the cylinder.

The scattered pressure field is usually evaluated in the far-field $(r \gg a)$ at a fixed angle $\theta$ for a range of frequencies. The resulting far-field amplitude spectrum, which is called the form function is obtained from the following equation ${ }^{7}$

$$
f_{\infty}(\theta, k a)=\left(\frac{2 r}{a}\right)^{1 / 2} p_{s} e^{-i k_{\perp} r},
$$

where $k a$, the product of wave number and cylinder radius, is the normalized frequency. The form function can be written as the sum of individual normal modes,

$$
f_{\infty}(\theta, k a)=\sum_{n=0}^{\infty} f_{n}(\theta, k a)=\sum_{n=0}^{\infty} \frac{2}{\sqrt{i \pi k_{\perp} a}} \epsilon_{n} A_{n} \cos (n \theta) .
$$

Resonance scattering theory (RST) states that the spectrum of the returned echo consists of two distinct parts: the first part varies smoothly with frequency and would be present even if the cylinder were impenetrable (nonresonant background), and the other part is the resonance spectrum which consists of a number of resonance peaks which coincide with the eigenfrequencies of the circumferential vibrations of the cylinder.

For a cylindrical geometry, the nonresonant background scattering component (rigid background in case of metal cylinders) can be separated from the resonance scattering part. For a rigid cylinder the scattering coefficients $A_{n}^{\text {(rigid) }}$ are $^{7}$

$$
A_{n}^{(\text {rigid })}(k a)=-\frac{J_{n}^{\prime}(k a)}{H_{n}^{(1)^{\prime}}(k a)} .
$$

The resonance spectrum of each mode can be obtained by removing the rigid background, ${ }^{26}$

$$
f_{n}^{(\text {res })}(\theta, k a)=\frac{2}{\sqrt{i \pi k_{\perp} a}} \epsilon_{n} \frac{A_{n}-A_{n}^{\text {(rigid) }}}{1+2 A_{n}^{\text {(rigid) }}} \cos (n \theta) .
$$

The displacement field inside the cylinder can also be determined by solving Eq. (8) for $B_{n}, C_{n}$ and $D_{n}$ and substituting these values in Eq. (3).

\section{EFFECT OF ANGLE OF INCIDENCE ON RESONANCE MODES}

As mentioned earlier, for a solid elastic cylinder, the form function consists of resonance spectra superimposed on a smooth background corresponding to a rigid cylinder. The resonance modes in the spectrum are linked to the standing surface waves which are formed around the cylinder. The surface waves are divided into three major types: pseudoRayleigh waves, whispering gallery waves and axially guided waves. The axially guided waves only appear when the incident wave angle is nonzero, i.e., $\alpha \neq 0$.

Figure 2 shows the form function of an aluminum cylinder insonified by an obliquely incident plane acoustic wave at $\alpha=5^{\circ}$. Each dip in Fig. 2 is due to a certain resonance frequency identified by the integers $(n, l)$. The first of these two integers defines the mode number and the second one indicates the eigenfrequency label for that mode; $l=1$ corresponds to a pseudo-Rayleigh wave and $l=2,3, \ldots$ to whispering gallery waves. Resonances associated with axially guided waves are designated by $\langle n, p\rangle$ where, $n$ is the mode number and $p$ is the eigenfrequency label.

Figure 3 shows the resonance frequencies of an immersed aluminum cylinder insonified by a plane acoustic wave for $0^{\circ} \leqslant \alpha \leqslant 40^{\circ}$. These resonances correspond to frequencies for which the real part of the determinant of the coefficient matrix in Eq. (8) goes to zero. ${ }^{7}$ (Typical ratios of imaginary to real components of the wave number are less than $10^{-4}$. Considering this small ratio, it is an acceptable approximation to ignore the imaginary component when calculating the resonance frequencies.) Figures 3(a)-3(d) show the variation of frequencies of resonance modes with changes in the incidence angle for different resonance modes. Key resonance modes are labeled according to their $(n, l)$ or $\langle n, l\rangle$ designation in Fig. 3(a).

An extension to the information conveyed by Fig. 3 can be displayed by plotting the resonance curves using Eq. (12). Figure 4 shows the resonance spectrum of the aluminum cylinder corresponding to $\alpha=5^{\circ}$. The advantage of using Eq. (12) is that both the center frequency and bandwidth (all information contained in the complex frequency) of each resonance mode are shown in Fig. 4; hence enabling the comparison of their attenuations. In Fig. 5, we have used the resonance spectra obtained from Eq. (12) at different incidence angles for plotting the resonance curves. Figure 5 is a top view of resonance curves plotted at different incident angles and placed next to one another. The bandwidth of the resonance frequencies is indicated by the thickness of the dark lines describing the curves. It can be observed that although different approaches are used for plotting Figs. 3(a) and 5(a), they both show the same characteristic behavior of the resonance curves.

\section{A. Shift of the resonance frequencies}

Figures 3 and 5 show the changes in frequencies of specific resonance modes of an aluminum cylinder with increase 


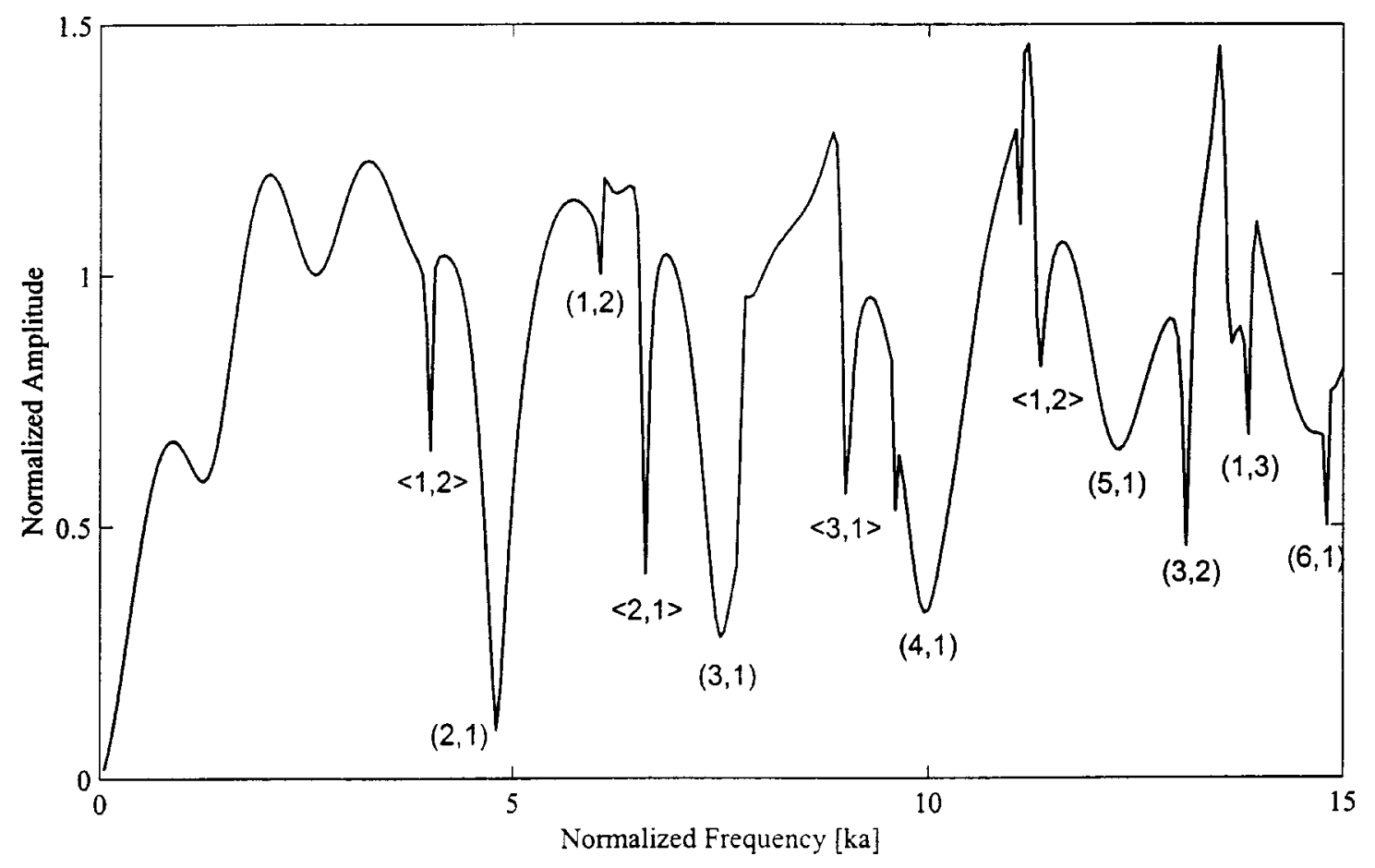

FIG. 2. Form function for an aluminum cylinder at $\alpha=5^{\circ}$.
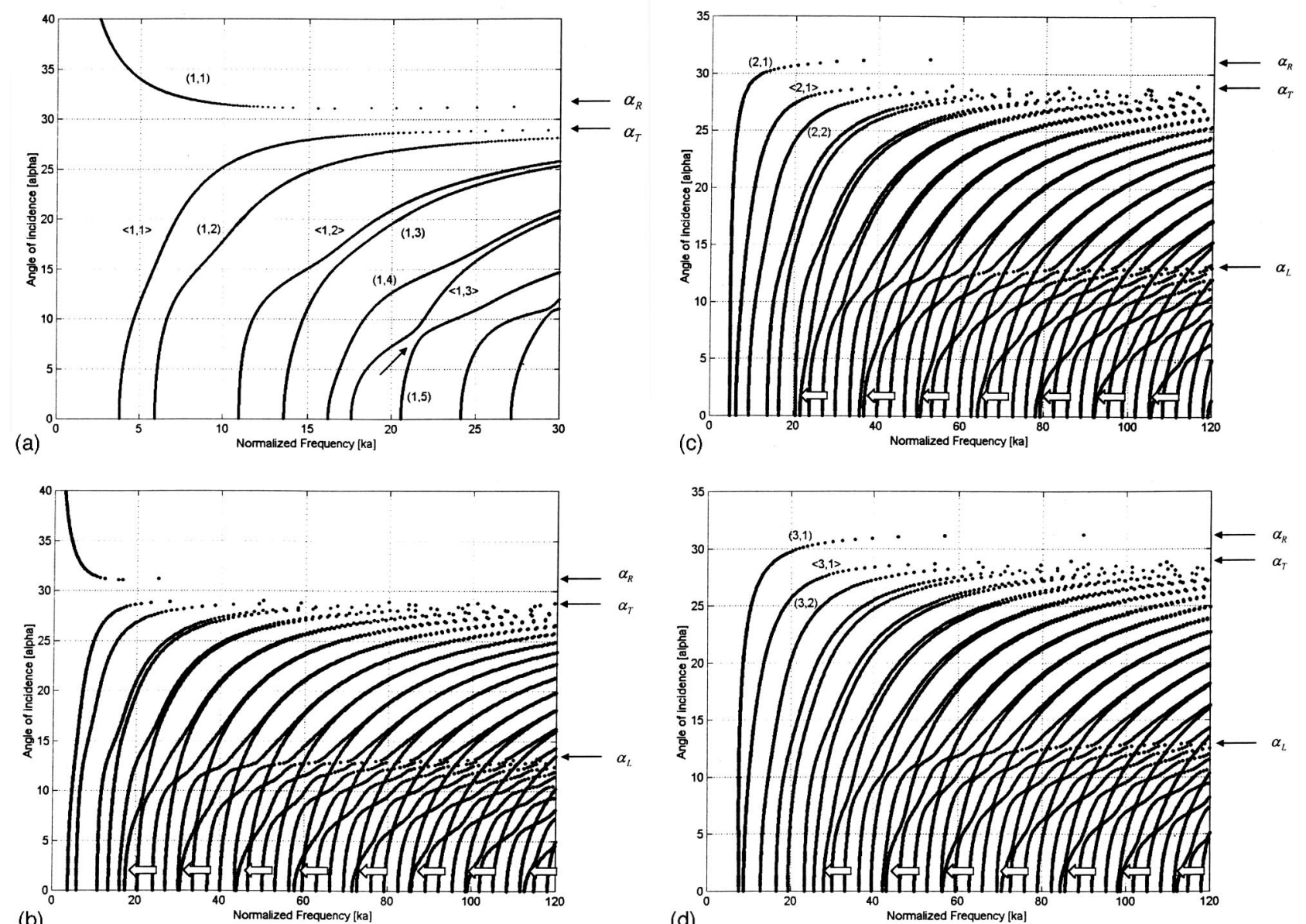

(b)

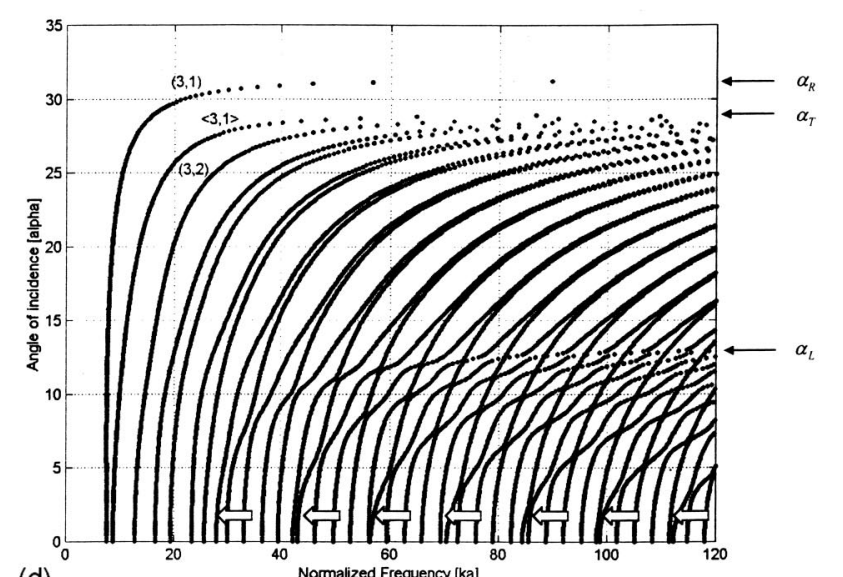

(d)

FIG. 3. Resonance curves of an aluminum cylinder. The resonance frequencies are the roots of the real part of the coefficient matrix of Eq. (8). (a) $n=1$ and $0 \leqslant k a \leqslant 30$, (b) $n=1$ and $0 \leqslant k a \leqslant 120$, (c) $n=2$, (d) $n=3$. 


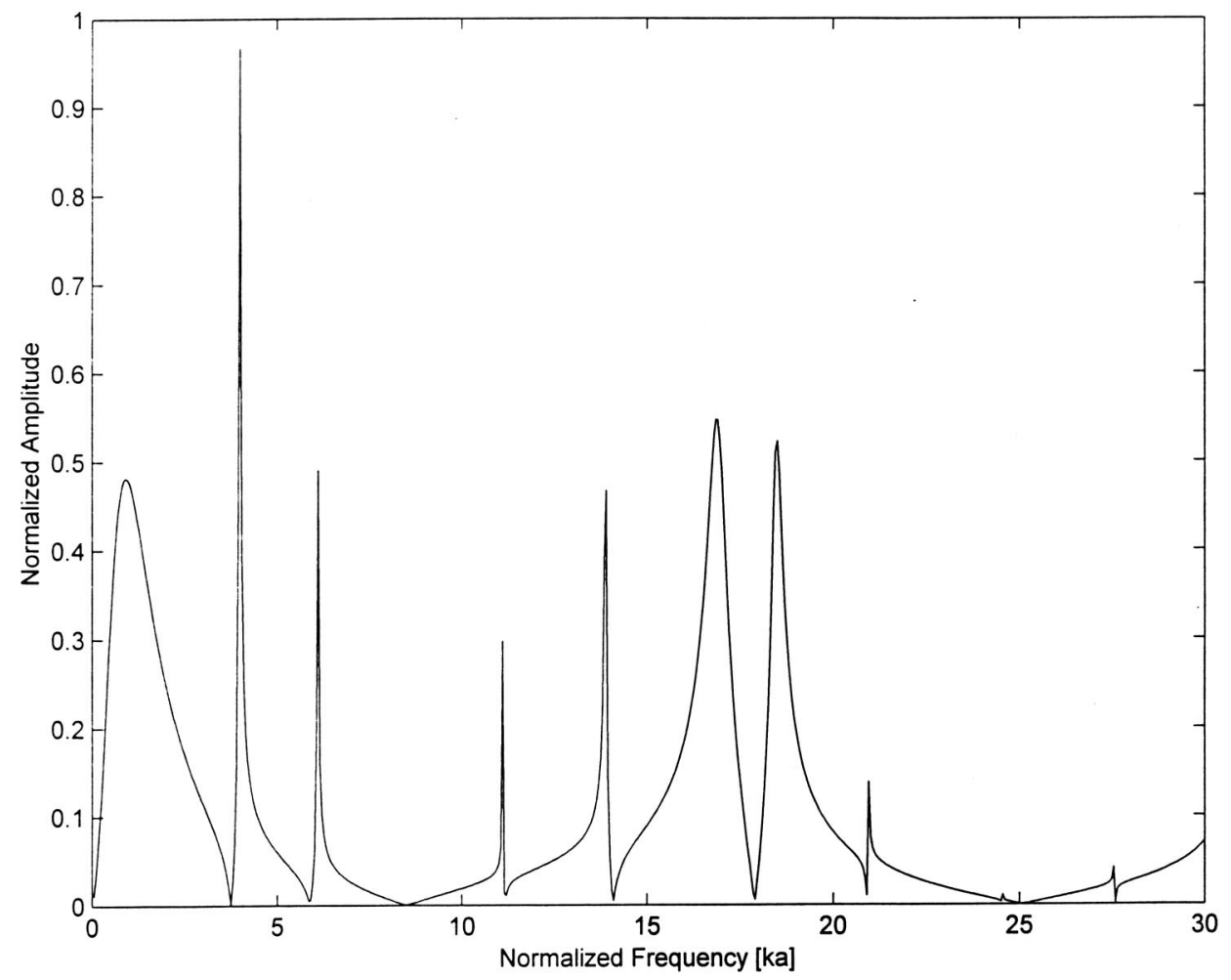

FIG. 4. Resonance spectrum of an aluminum cylinder at $\alpha=5^{\circ}$.

of the angle of incidence. It is observed that below the second critical angle, $\alpha_{T}$, all the resonance frequencies corresponding to whispering gallery and axially guided waves increase monotonically with the increase of $\alpha$, and tend to infinity as $\alpha$ approaches the second critical angle, $\alpha_{T}$. A similar behavior is observed for pseudo-Rayleigh waves with higher mode numbers $(n \geqslant 2$, and $l=1)$; see Figs. 3(c) and (d). The pseudo-Rayleigh mode corresponding to $n=1$ in Figs. 3(a) and (b) is special and will be considered in the next section. With increase of the angle of incidence, pseudoRayleigh waves approach the true Rayleigh wave (on a submerged plate) and the corresponding resonance frequencies approaches the Rayleigh angle, $\alpha_{R} \simeq 31^{\circ}$.

A similar observation was reported by Conoir et al., ${ }^{24}$ who explained the shift of resonance frequencies using the connection between the propagation of helical surface waves around the cylinder and formation of resonance modes.

Following the approach of Ref. 24, a physical explanation for the rightward shift of all resonance frequencies with an increase in the angle of incidence can be presented as follows. In physical terms, a resonance indicates a standing wave pattern. For the case of a normally incident wave, an integral number $n$ of wavelengths of the corresponding wave pattern are distributed around the circumference of the cylinder such that,

$$
\lambda_{n}=2 \pi a / n \text { and } k_{n}=2 \pi / \lambda_{n},
$$

where $\lambda_{n}$ and $k_{n}$ are the wave number and the projection of the total wave vector in the $\theta$ direction, respectively. For an obliquely incident wave, $k_{n}$ can be related to the total wave vector $k_{\gamma}$ by (see Fig. 6),

$$
k_{n}=k_{\gamma} \cos \gamma=2 \pi \cos \gamma / \lambda_{\gamma}
$$

where $\gamma$ is the refraction angle and $\lambda_{\gamma}$ is the wavelength. Substituting Eq. (13) into Eq. (14), $\lambda_{n}$ can be related to the helical wavelength $\lambda_{\gamma}$ by

$$
\lambda_{\gamma}=\lambda_{n} \cos \gamma
$$

The resonance frequency is

$$
f_{\text {res }}=c_{p h} / \lambda_{\gamma},
$$

where $c_{p h}$ is the phase velocity of the corresponding surface wave. Substituting Eqs. (13) and (15) into Eq. (16) gives

$$
f_{\text {res }}=\left(n c_{p h}\right) /(2 \pi a \cos \gamma) \text {. }
$$

Equation (17) shows that the resonance frequency is inversely proportional to $\cos \gamma$. For the case of immersed elastic cylinders, an increase in $\alpha$ causes an increase of the refraction angle $\gamma$ and consequently a decrease in $\cos \gamma$. Therefore, the resonance frequency $f$ increases with the increase of the angle of incidence, $\alpha$. When $\gamma$ reaches $\pi / 2$ (i.e., critical angle), the resonance frequency tends to infinity.

\section{B. Resonance mode $(1,1)$}

A single pseudo-Rayleigh wave corresponding to mode ( $n, 1)$ is present at $\alpha \leqslant \alpha_{R}$ when $n>1$. The corresponding (2, $1)$ and $(3,1)$ modes for an immersed aluminum cylinder are shown in Figs. 3(c) and (d), respectively.

The $(1,1)$ resonance mode, shown in Figs. 3(a) and 5(a) is anomalous. Curiously, this pseudo-Rayleigh mode for $n$ $=1$ appears only for incident angles greater or equal to $\alpha_{R}$, such that one would expect that penetration of energy into the cylinder would be impossible. The existence of resonances above Rayleigh angle is reported in the literature, ${ }^{27}$ however, no physical explanation is provided. To confirm the 

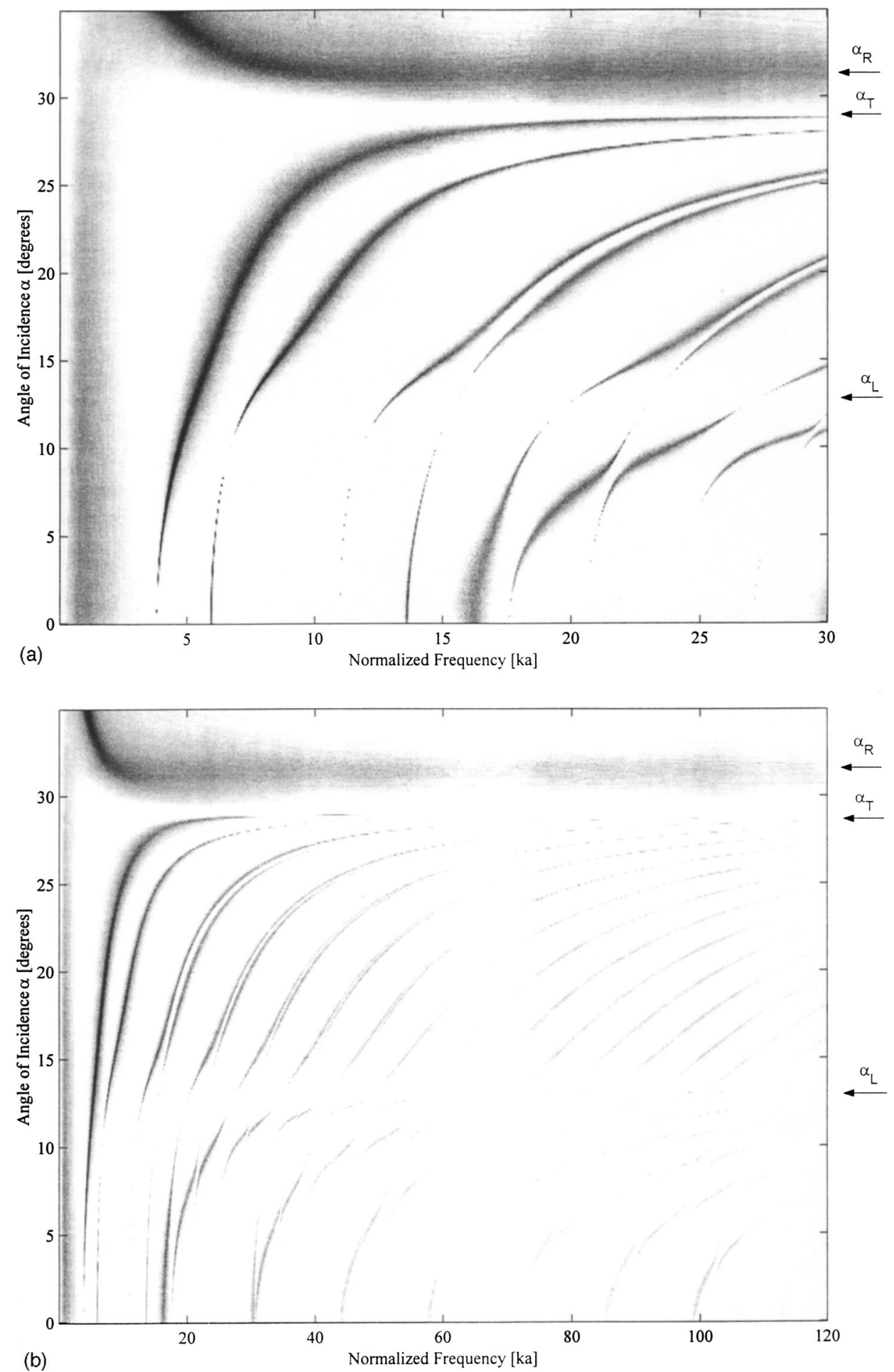

FIG. 5. Resonance curves of an aluminum cylinder. This is the top view of a three dimensional graph obtained by plotting the resonance spectra for different incident angles using Eq. (12). (a) $n=1$ and $0 \leqslant k a \leqslant 30$, (b) $n=1$ and $0 \leqslant k a \leqslant 120$. existence of this resonance mode, plots showing the amplitude and phase changes at resonance frequencies corresponding to various incident angles are plotted in Fig. 7 based on Eq. (12) for $\alpha=30^{\circ}$ and $\alpha=35^{\circ}$. The existence of a resonant mode beyond the second critical angle is indicated in the phase diagram by a sudden phase shift; see Fig. 7(d).

The pseudo-Rayleigh mode $(1,1)$ is alternatively referred to as the rigid-body translation mode, i.e., the cylinder moves vertically back and forth without distortion. Figure 8(a) shows the displacement field at a cross-section of the cylinder for the $(1,1)$ mode at $\alpha=33^{\circ}$. It is observed that the true motion is made up primarily of a common translational component. Figure 8(b) shows the displacement field for the same mode at $k a \simeq 67$ and $\alpha=31.24^{\circ}$. The corresponding displacement field for higher order pseudo-Rayleigh modes, e.g., $(2,1)$ and $(3,1)$, at comparable $k a$ values showed a similar displacement field, hence, indicating that all pseudoRayleigh modes approach the true Rayleigh mode at high frequencies. The reason for the upward curving of $(1,1)$ mode with the increase of $\alpha$ is not completely understood yet 


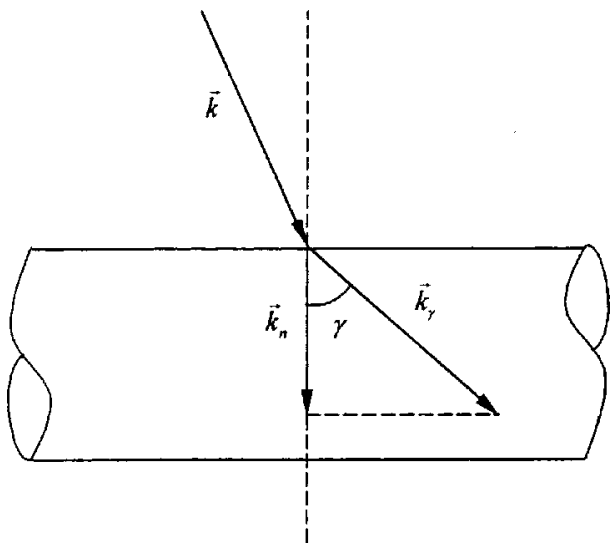

FIG. 6. Refracted surface wave on the cylinder.

but it can be correlated to corresponding decrease in the radial component of the wave vector. In other words, if the wavelength is larger than a certain value, this mode cannot be directly generated.

The pressure amplitude, $\sigma_{r r}$, at the boundary of the cylinder is plotted for mode $(1,1)$ in Fig. 9. As shown in Fig. 9, $\sigma_{r r}$ is zero at $\theta=90^{\circ}$ and $\theta=270^{\circ}$ and it reaches its maximum value at $\theta=0^{\circ}$ and $\theta=180^{\circ}$, which indicates that an external force acts in a horizontal direction on the cylinder and causes a translational motion, see the arrows next to the cylinder in Fig. 9.

\section{Compressional whispering gallery waves}

There exist two types of whispering gallery waves. The waves in the first category are called transverse whispering gallery waves, which feature predominantly shearing stresses. A qualitative description of the ray paths for these waves was presented by Uberall. ${ }^{28}$ The wave vectors point along chords that link two points on the boundary of the cylinder. The number of wavelengths within each chord, determines the resonance mode number. A detailed mathematical analysis was given by Brekhovskikh, ${ }^{29}$ and by Dickey et $a l .{ }^{30}$ In the limit of large values of $k a$, the wave speed approaches the bulk shear wave velocity.

The second category of whispering gallery waves features predominantly normal stresses. Unlike their shearing counterparts, the imaginary parts of these compressional whispering gallery resonances are quite dominant and consequently they are significantly attenuated. For this reason, such resonances are not observed experimentally. ${ }^{30}$

All of the surface waves corresponding to resonance modes shown in Figs. 3 and 5 feature predominantly shearing actions. The imaginary parts of their frequencies are small giving them very small attenuations. For example, for the resonance frequencies shown in Fig. 3(a), the maximum ratio of the imaginary to real part is in the range of $10^{-4}$. Considering the transverse nature of these resonance modes, one would expect that the first critical angle would not play a major role in the values of resonance frequencies shown in Fig. 3. Examination of Fig. 3, however, shows that some curious features are observed at incident angles $0 \leqslant \alpha \leqslant \alpha_{L}$. These features are:
(1) Severe serpentine distortions are noted in the $k a-\alpha$ plots for both the transverse whispering gallery modes as well as the guided modes within this range of low $\alpha$ values. These distortions are not random; in several locations, there is a marked deviation of two different modes towards a specific point. A typical example is indicated by the solid arrow in Fig. 3(a), where both the $\langle 1,3\rangle$ and $(1,5)$ modes deviate toward the point $(\alpha$ $\left.=8.6^{\circ}, k a=22\right)$.

(2) The distortions described in item (1) form distinct patterns that are clearly visible in Figs. 3 and 5. The patterns consist of arcs rising vertically from the horizontal axis, then curving to the right, and then asymptotically approaching the line $\alpha=\alpha_{L}$ at high frequencies. Some of the intercepts of these arcs with the horizontal axis are indicated by block arrows in Figs. 3(b), (c), and (d).

The origin of arc patterns mentioned above was numerically determined to be the leaky compressional whispering gallery modes. At the complex frequencies corresponding to these modes, the determinant of the real part of the coefficient matrix in Eq. (8) goes to zero. However, previous work by Dickey et $a l .{ }^{30}$ showed that it is extremely difficult to find these particular complex roots corresponding to oblique incidence because a large number of roots are clustered close together. Despite the fact that these modes are not explicitly featured in Fig. 3, their influence is still present, i.e., resonance frequencies of guided and transverse whispering gallery modes are significantly distorted when they are located close to one of the leaky compressional whispering gallery modes. As a result, the locus of resonances corresponding to each compressional whispering gallery mode appears as an arcing shadow in Figs. 3(b), (c), and (d).

To verify this conclusion, based on the shadow arcs, dispersion curves for the first three compressional whispering gallery modes are plotted corresponding to normal incidence $(\alpha=0)$. For this purpose, we use the relation $c_{p h}$ $=(2 \pi a f) / n$, where $f$ is the resonance frequency in $\mathrm{Hz}$ and is derived from the $k a$ values observed in Figs. 3(b), (c), and (d). These $k a$ values are the normalized frequencies corresponding to the first three shadow arcs at $\alpha=0^{\circ}$ in each figure. Figure 10 shows these dispersion curves, which agree with the results reported by Dickey et al. ${ }^{30}$ In the limit of high radius-to-wavelength ratio, the speed of longitudinal whispering gallery waves approaches the speed of the longitudinal lateral waves. As a result, the shadow arcs in Fig. 3 are strongly dependent on the axial stiffness of the rod; their shapes could therefore be used to help assess the extent of anisotropy in transversely isotropic cylinders.

It is noteworthy that an analogous behavior has been observed by Uberall et al. in the study of the dispersion curves of Lamb waves propagating in elastic plates. ${ }^{31}$ The dispersion curves corresponding to symmetric and antisymmetric modes of Lamb waves show very similar serpentine distortions. This behavior, which is very similar to that observed in our study is referred to as "repulsion of the curves" in Ref. 31. This similarity is consistent with the dispersive nature of guided waves in solid cylinders and plates. ${ }^{32}$ 

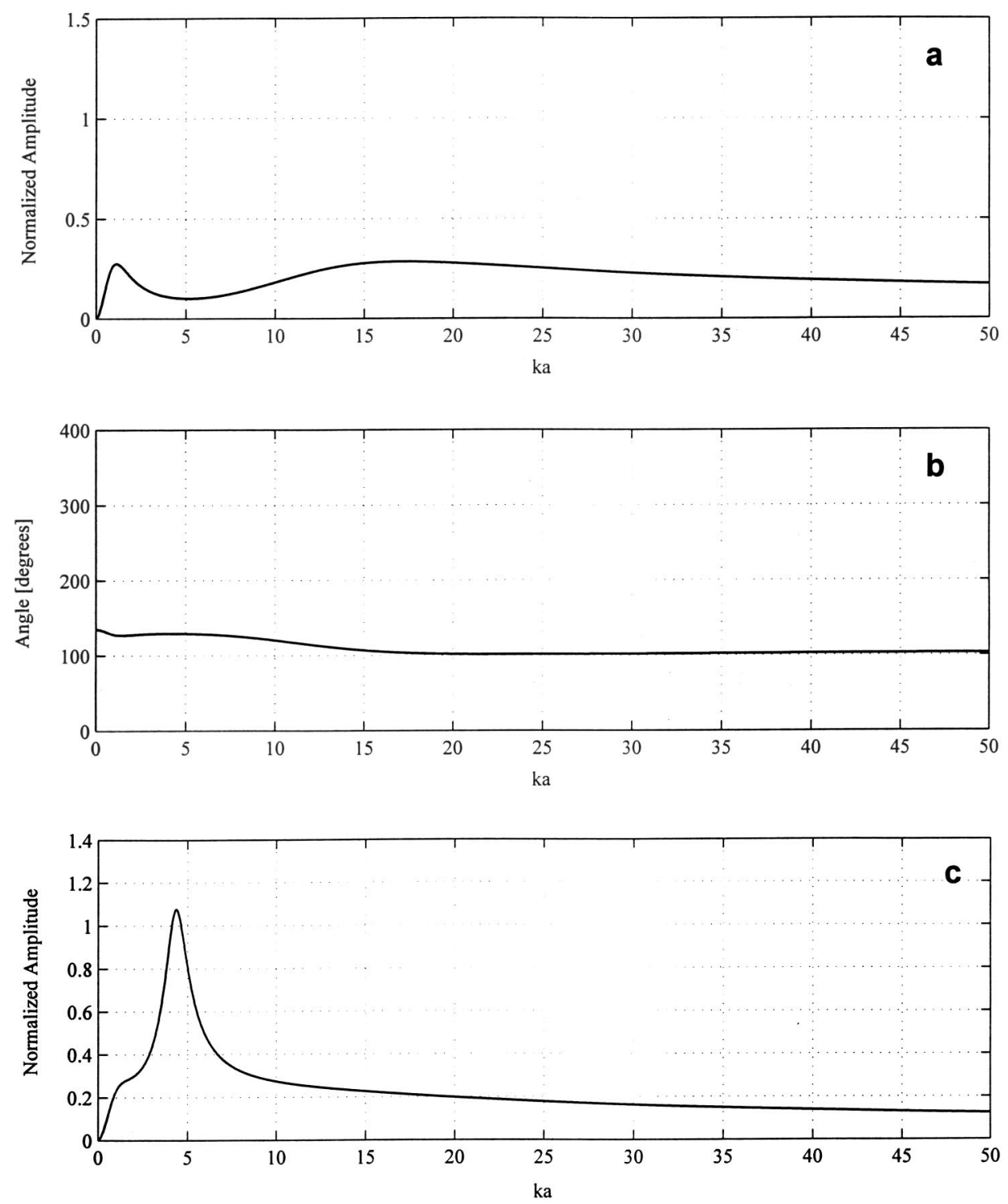

FIG. 7. Resonance mode $(1,1)$ : (a) amplitude spectrum at $\alpha=30^{\circ}$, (b) phase diagram at $\alpha=30^{\circ}$, (c) amplitude spectrum at $\alpha=35^{\circ}$, (d) phase diagram at $\alpha=35^{\circ}$.

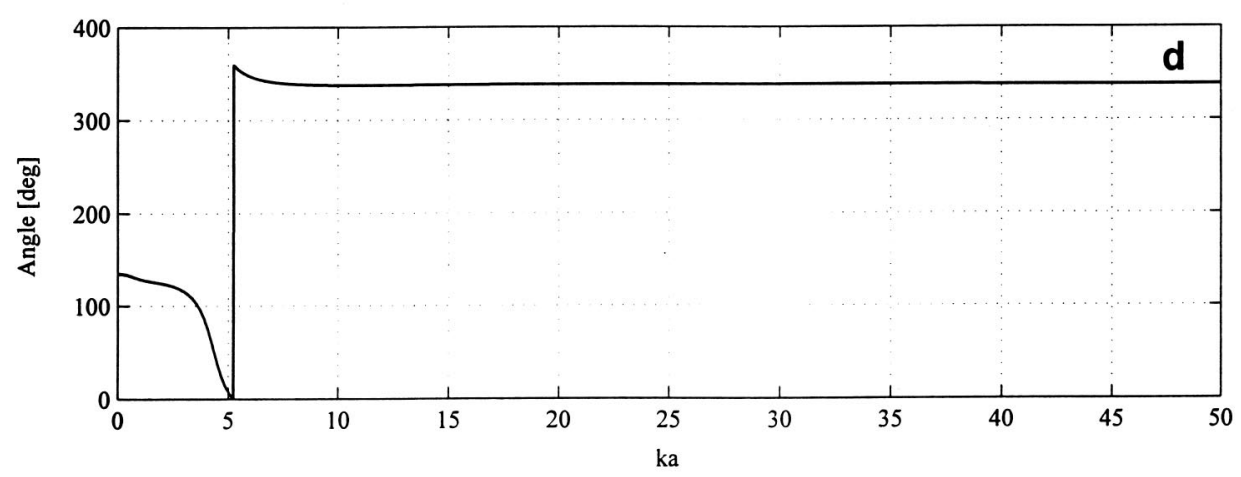

\section{Wave pairs}

As seen in Fig. 3, each transverse whispering gallery mode is paired with a single guided wave mode, such that the two modes become indistinguishable as $\alpha$ approaches $\alpha_{T}$. For small values of $\alpha$, each mode pair splits in two. When $\alpha=0$, the guided mode disappears completely, leaving only the transverse whispering gallery component of each pair.

The merging of these two modes at large incident angles can be explained by considering the physics of transverse whispering gallery and axially guided modes. Both modes feature primarily shear action. The axially guided waves propagate in a helical path along the axial direction of the cylinder. The angle of each helix depends on the incidence angle and phase velocity of the corresponding surface wave. It has been shown by Dickey et al. ${ }^{30}$ that in the limit when the cylinder radius tends to infinity, the displacement fields of the transverse/longitudinal whispering gallery modes be- 

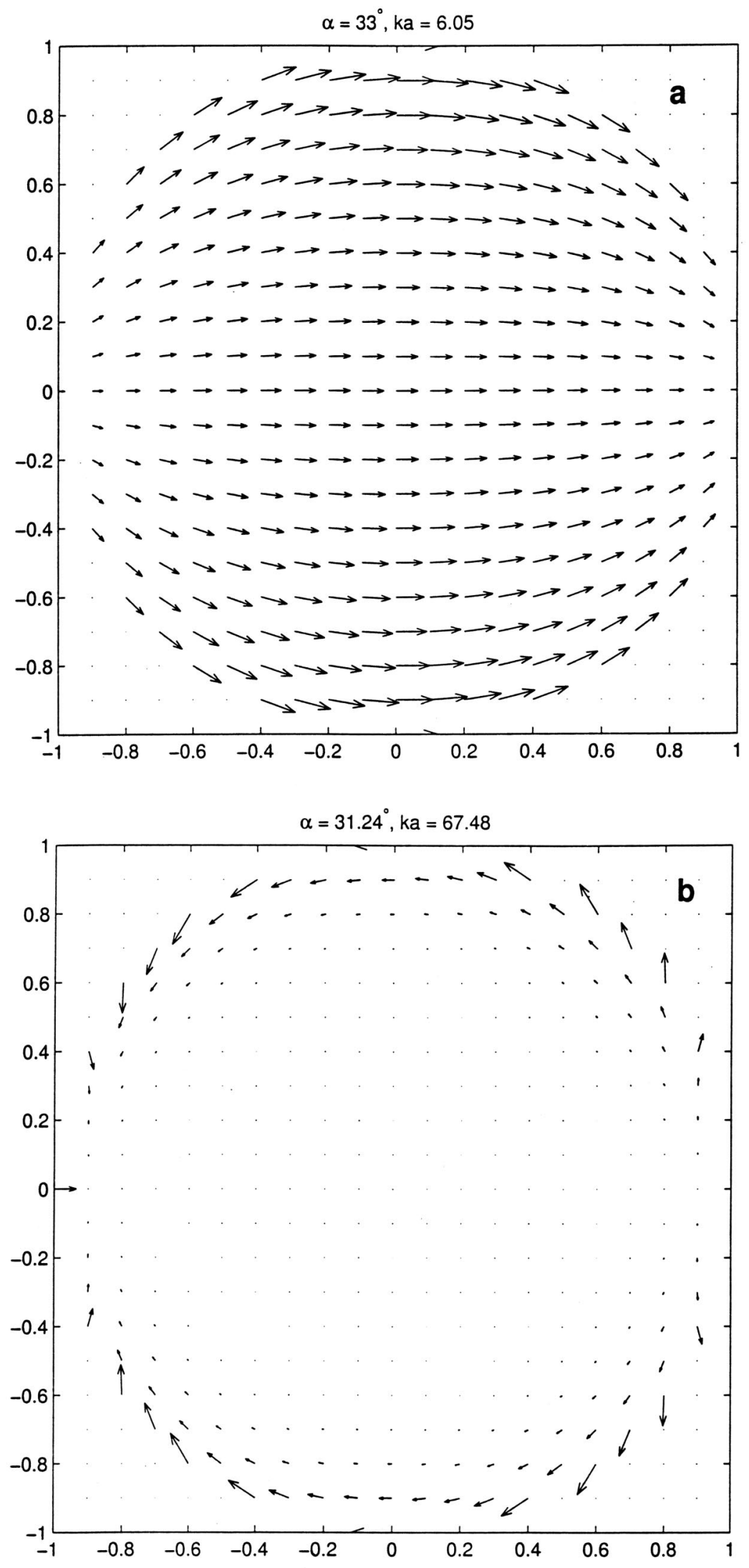

FIG. 8. (a) Displacement field at a cross section of the aluminum cylinder for the $(1,1)$ mode at $\alpha=33^{\circ}$ and $k a=6.05$. The length of the arrow is proportional to the amplitude of the displacement. (b) Displacement field at a cross section of the aluminum cylinder for the $(1,1)$ mode at $\alpha=\alpha_{R}$ and $k a \simeq 67$. come the expressions for the transverse/longitudinal lateral waves on a flat elastic half-space bounded by a fluid. As the incident angle increases, the whispering gallery waves tend to propagate close to the surface and along the axial direction of the cylinder, in a manner very similar to an axially guided wave. These two types of modes merge into one as the incident angle approaches the second critical angle. To confirm this point, two sets of resonance modes corresponding to 

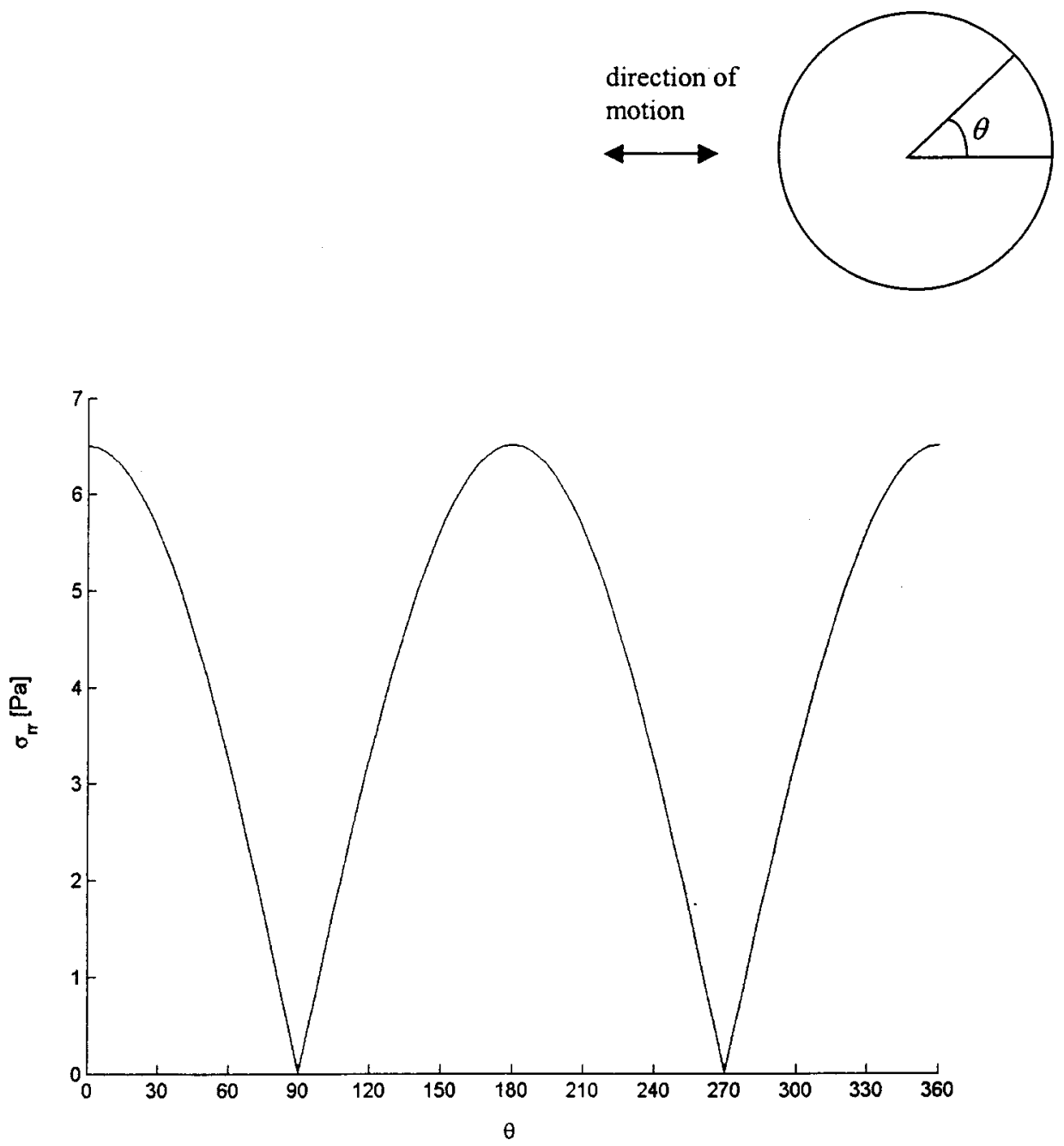

FIG. 9. Stress $\sigma_{r r}$ at the boundary of the cylinder for mode $(1,1)$, the transmitter is located at $\theta=180^{\circ}$.

different incidence angles are selected in Fig. 3(a) and the displacement field of each mode is plotted in Fig. 11. Within each pair of modes, one corresponds to the axially guided mode $\langle 1,2\rangle$ and the other to the whispering gallery mode (1,
3). At the small incidence angle of $\alpha=5^{\circ}$, as shown in Fig. 11(a), these two modes have different displacement fields. As the incidence angle approaches $\alpha_{T}$, the corresponding displacement fields $u_{z}$ and $u_{\theta}$ of the two modes become identi-

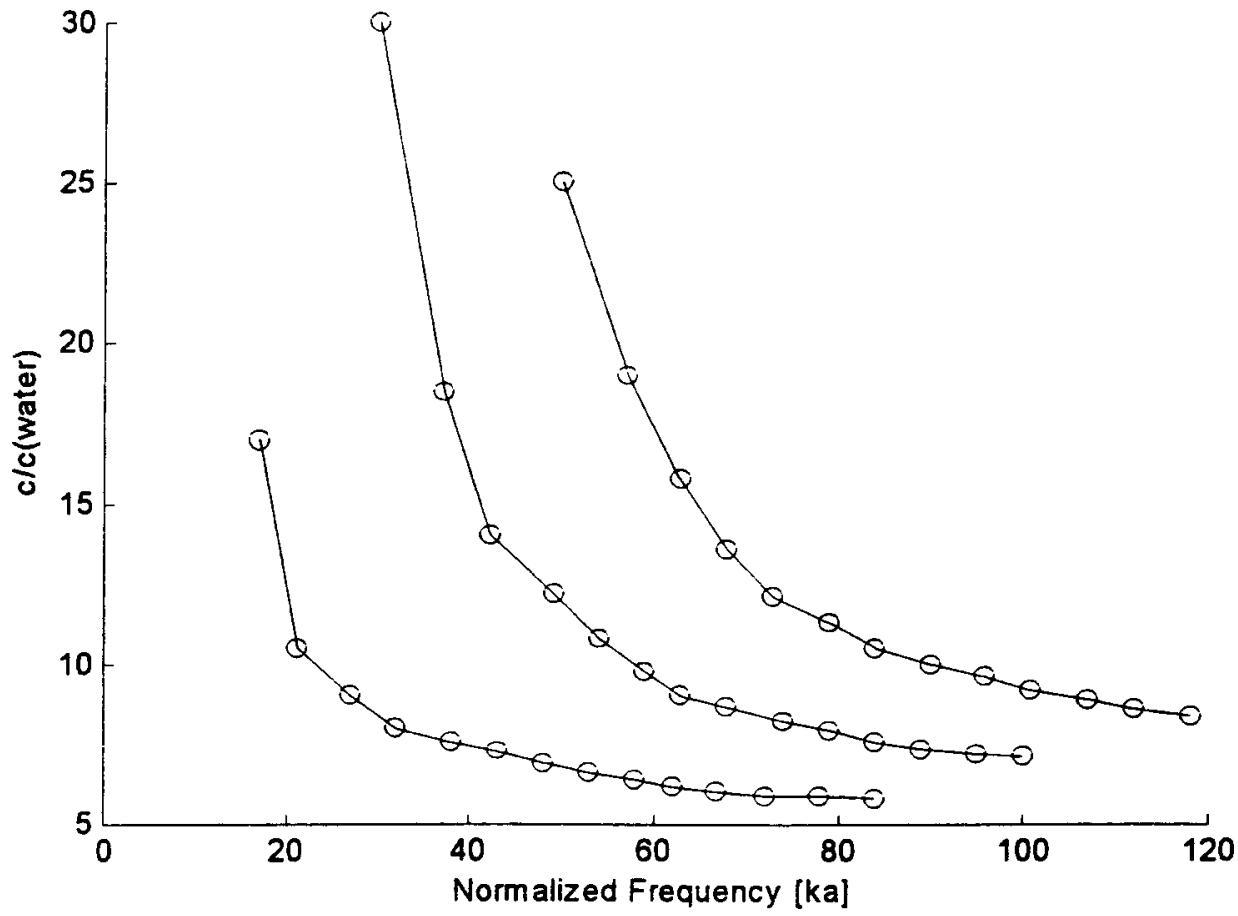

FIG. 10. Dispersion curves for the first three groups of longitudinal whispering gallery waves. 


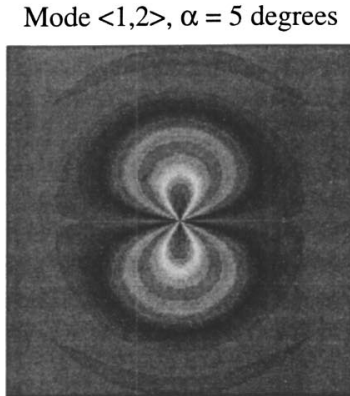

$\mathrm{u}_{\mathrm{r}}$

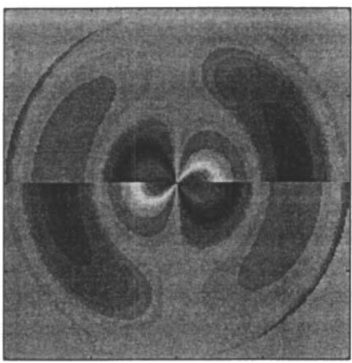

$u_{\theta}$

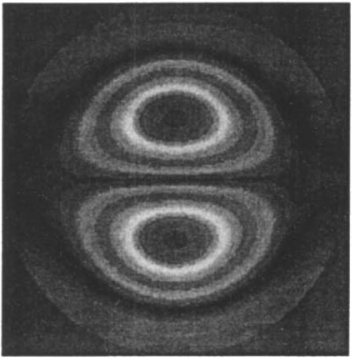

$\mathrm{u}_{\mathrm{z}}$
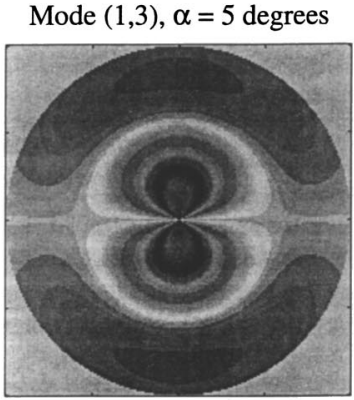

$u_{r}$

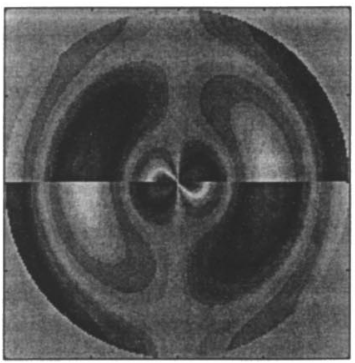

$\mathbf{u}_{\theta}$

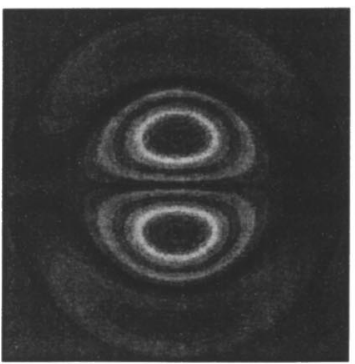

$\mathrm{u}_{\mathbf{z}}$

(a)

FIG. 11. (a) Displacement fields for mode $\langle 1,2\rangle$ and $(1,3)$ at $\alpha=5^{\circ}$
Mode $<1,2>, \alpha=25.81$ degrees

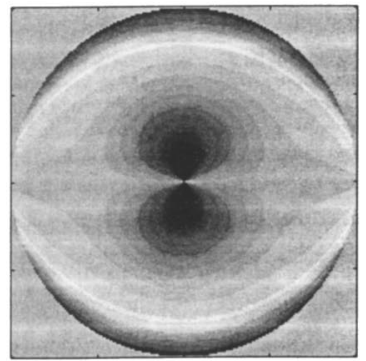

$u_{r}$

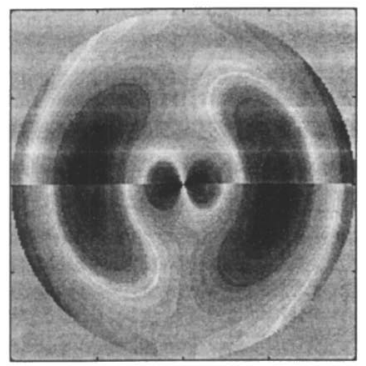

$\mathrm{u}_{\theta}$

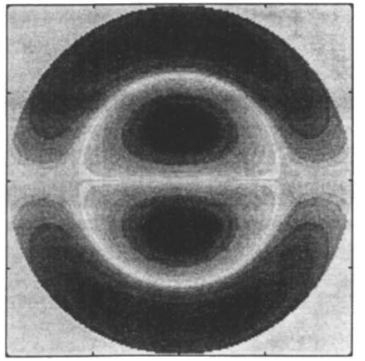

$\mathrm{u}_{\mathrm{z}}$
Mode $(1,3), \alpha=25.34$ degrees

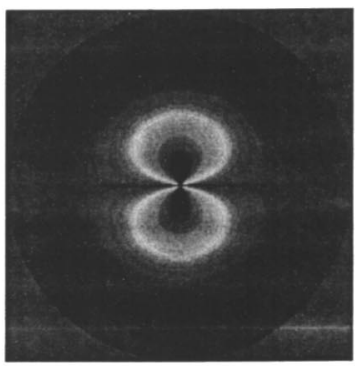

$\mathrm{u}_{\mathrm{r}}$

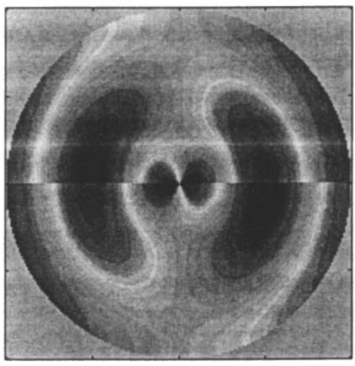

$u_{\theta}$

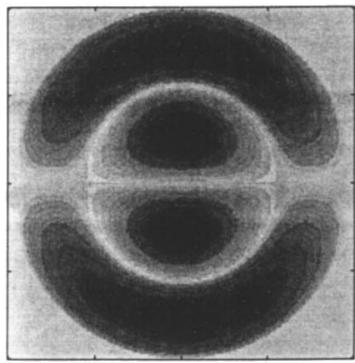

$\mathrm{u}_{\mathrm{z}}$

(b)

(b) Displacement fields for mode $\langle 1,2\rangle$ and $(1,3)$ at $\alpha \simeq 25^{\circ}$.

cal, see Fig. 11(b), while $u_{r}$ fields, although equal in magnitude, show opposite directions. Consequently, at large incidence angles, radial displacement fields of these two waves cancel each other. Therefore, the merging of a whispering gallery wave and its adjacent axially guided wave, at large incidence angles, results in a guided wave propagating along the axis of the cylinder without any radial components.

\section{CONCLUSIONS}

Using Resonance Scattering Theory (RST), the resonance modes of an immersed aluminum cylinder were studied over a large range of incidence angles. With the increase of the incidence angle, it was observed that the resonance frequencies shift to higher frequencies in a nonuniform manner. The distortion of the $k a-\alpha$ curves at incidence angles below the first critical angle can be attributed to modes associated with leaky compressional whispering gallery waves. As the angle of incidence approaches the second critical angle, resonance frequencies of both whispering gallery and axially guided modes tend to infinity, while resonance frequencies of pseudo-Rayleigh modes do not tend to infinity, until incidence angle is close to the Rayleigh angle. The (1, 1) pseudo-Rayleigh mode behave differently and only appears at or above the Rayleigh angle. The motion of this resonance mode is made up primarily of a common translational component and resembles the true Rayleigh wave on a submerged cylinder. The whispering gallery and axially guided modes observed in the $k a-\alpha$ plots feature predominantly shear action. At large incidence angles, a whispering gallery mode and its neighboring axially guided mode merge together and produce similar but opposite radial displacement fields such that the resultant wave does not have any radial components.

\footnotetext{
${ }^{1}$ F. Honarvar and A. N. Sinclair, "Nondestructive evaluation of cylindrical components by resonance acoustic spectroscopy," Ultrasonics 36, 845854 (1998).

${ }^{2}$ F. Honarvar and A. N. Sinclair, "Scattering of an obliquely incident plane wave from a circular clad rod," J. Acoust. Soc. Am. 102, 1-8 (1997).

${ }^{3}$ J. J. Faran, Jr., "Sound Scattering by solid cylinders and spheres," J. Acoust. Soc. Am. 23, 405-418 (1951)

${ }^{4}$ Visual Numerics, Inc., IMSL C Numerical Library, 1991

${ }^{5}$ The Mathworks, Inc., Using Matlab, 2000.

${ }^{6}$ L. Flax, L. R. Dragonette, and H. Überall, "Theory of resonance excita-
} 
tion by sound scattering," J. Acoust. Soc. Am. 63, 723-731 (1978).

${ }^{7}$ L. Flax, G. C. Gaunaurd, and H. Überall, Theory of Resonance Scattering, Vol. 15 of Physical Acoustics (Academic, New York, 1981), pp. 191-294, Chap. 3.

${ }^{8}$ H. Überall, Acoustic Resonance Scattering (Gordon and Breach Science, Philadelphia, PA, 1992).

${ }^{9}$ L. Flax, V. K. Varadan, and V. V. Varadan, "Scattering of an obliquely incident acoustic wave by an infinite cylinder," J. Acoust. Soc. Am. 68, 1832-1835 (1980).

${ }^{10} \mathrm{~T}$. Li and M. Ueda, "Sound scattering of a plane wave obliquely incident on a cylinder," J. Acoust. Soc. Am. 86, 2363-2367 (1989).

${ }^{11}$ N. D. Veksler, Resonance Acoustic Spectroscopy, Springer Series on Wave Phenomena (Springer-Verlag, New York, 1993).

${ }^{12}$ G. V. Frisk, J. W. Dickey, and H. Überall, "Surface wave modes on elastic cylinders," J. Acoust. Soc. Am. 58, 996-1008 (1975).

${ }^{13}$ L. Flax and W. G. Neubauer, "Acoustic reflection from layered absorptive cylinders," J. Acoust. Soc. Am. 61, 307-312 (1977).

${ }^{14}$ R. C. Addison, Jr. and A. N. Sinclair, "Calculated and Measured Ultrasonic Response of an Elastic Cylinder Embedded in an Elastic Medium," in Review of Progress in Quantitative Nondestructive Evaluation, edited by D. O. Thompson and D. E. Chimenti (Brunswick, Maine, 1991).

${ }^{15}$ Y. Fan, A. N. Sinclair, and F. Honarvar, "Scattering of a plane acoustic wave from a transversely isotropic cylinder encased in a solid elastic medium," J. Acoust. Soc. Am. 106, 1229-1236 (1999).

${ }^{16}$ F. Honarvar and A. N. Sinclair, "Acoustic wave scattering from transversely isotropic cylinders," J. Acoust. Soc. Am. 100, 57-63 (1996).

${ }^{17}$ F. Ahmad and A. Rahman, "Acoustic scattering by transversely isotropic cylinder,” Int. J. Eng. Sci. 38, 325-335 (2000).

${ }^{18}$ G. C. Gaunaurd, "Elastic and acoustic resonance wave scattering," Appl. Mech. Rev. 42, 143-192 (1989).

${ }^{19}$ A. Ohno, "Continuous-casting of single-crystal ingots by the OCC process," J. Met. 38, 14-16 (1986).

${ }^{20}$ A. Sinclair, Y. Fan, and F. Honarvar, Ultrasonic Characterization of Con- tinuously Cast Rod by Resonance Acoustic Spectroscopy, Nondestructive Testing and Evaluation (Taylor and Francis, accepted).

${ }^{21}$ R. M. White, "Elastic wave scattering at a cylindrical discontinuity in a solid," J. Acoust. Soc. Am. 30, 771-785 (1958).

${ }^{22}$ A. Nagl, H. Überall, P. P. Delsanto, J. D. Almar, and E. Rosario, "Refraction effects in the generation of helical surface waves on a cylindrical obstacle," Wave Motion 5, 235-247 (1983).

${ }^{23}$ G. Maze, J. L. Izbicki, and J. Ripoche, "Resonances of plates and cylinders: Guided waves," J. Acoust. Soc. Am. 77, 1352-1357 (1985).

${ }^{24}$ J. M. Conoir, P. Rembert, O. Lenoir, and J. L. Izbicki, "Relation between surface helical waves and elastic cylinder resonances," J. Acoust. Soc. Am. 93, 1300-1307 (1993).

${ }^{25}$ F. Lecroq, G. Maze, D. Decultot, and J. Ripoche, "Acoustic scattering from an air-filled cylindrical shell with welded flat plate endcaps: Experimental and theoretical study," J. Acoust. Soc. Am. 95, 762-769 (1994).

${ }^{26} \mathrm{H}$. Rhee and Y. Park, "Novel acoustic wave resonance scattering formalism," J. Acoust. Soc. Am. 102, 3401-3412 (1997).

${ }^{27}$ J. M. Conoir, "Resonance scattering theory for oblique incidence: Scattering by an elastic infinite cylinder," in Electromagnetic and Acoustic Scattering: Detection and Inverse Problem Conference Proc. (Marseile, 1988), pp. 96-108.

${ }^{28}$ H. Überall, Surface Waves in Acoustics, Vol. 10 of Physical Acoustics (Academic, New York, 1973), Chap. 1.

${ }^{29}$ L. M. Brekhovskikh, "Surface waves confined to the curvature of the boundary in solids," Sov. Phys. Acoust. 13, 462-472 (1968).

${ }^{30}$ J. W. Dickey, G. V. Frisk, and H. Überall, "Whispering gallery wave modes on elastic cylinders," J. Acoust. Soc. Am. 59, 1339-1346 (1976).

${ }^{31} \mathrm{H}$. Uberall, B. Hosten, M. Deschamps, and A. Gerard, "Repulsion of phase-velocity dispersion curves and the nature of plate vibrations," J. Acoust. Soc. Am. 96, 908-917 (1994).

${ }^{32}$ J. L. Rose, Ultrasonic Waves in Solid Media (Cambridge University Press, New York, 1999). 